\title{
D evelopment and manufacture of an investigational human living dermal equivalent (ICX-SKN)
}

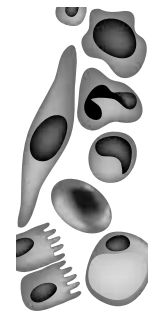

\section{Marzena Flasza ${ }^{1}$, Paul Kempit, David Shering ${ }^{1}$, Jizeng Q iao ${ }^{1}$, Damian Marshall2, Ausha Bokta ${ }^{1} \&$ Penny A Johnson ${ }^{1}$}

${ }^{\dagger}$ Author for correspondence IIntercytex Ltd., Innovation House, Crewe Road, $M$ anchester M 23 9QR, UK

Tel.: +44(0)1619044500 Fax: +44 (0)161 9044510

E-mail: pkemp@ intercytex.com 2 LGC, Q ueens Rd. Teddington,

TW 11 OLY, UK
Keywords autosynthesized human matrix, epithelialization, living skin equivalent, skin graft, tissue engineering and manufacture, wound healing

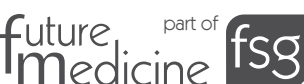

Aim: To design and manufacture an investigational living skin graft replacement (ICX-SKN) that is able to incorporate into the host, providing healing by primary intent without the need for a second intervention. Materials \& Methods: The ICX-SKN skin graft replacement has been designed as an allogeneic dermal substitute comprising an extracellular matrix composed largely of human collagen and human dermal fibroblast cells (HDFs). ICX-SKN is first formed by casting a provisional matrix of fibrin, into which HDFs are seeded. Through a process of maturation, HDFs are induced to lay down collagen and other extracellular matrix materials and, as the construct matures, the original fibrin is largely replaced by collagen, which provides tensile strength and flexibility to the construct. In order to design a product and manufacturing system that lends itself to largescale production the process was developed as a discontinuous process consisting of four stages: 1. batch casting and maturation of the initial construct (pSKN), 2. freeze-drying of pSKN to produce a second intermediate (dSKN), 3. sterilization by $\gamma$-irradiation of dSKN to produce a third intermediate (SSKN), and finally, 4. repopulation of SSKN by fresh HDFs to produce the final product, ICX-SKN skin graft replacement. Preliminary characterization of ICX-SKN and its application in a preclinical model are described. Results: The 7-week maturation period resulted in a construct (pSKN) with robust handling properties, which was composed mainly of human collagen I. Following development of a process for freezedrying and subsequent sterilization, the matrix was successfully repopulated with fresh HDFs. In addition, it was demonstrated that human keratinocytes attached and differentiated on the matrix. Application of human keratinocytes to the repopulated constructs (ICX-SKN) resulted in expression of markers of basement membranes that was largely dependent on the presence of living HDFs on the constructs. ICX-SKN graft replacements applied to excision wounds in mice healed and were rapidly re-epithelialized. Conclusions: ICX-SKN has been developed as a platform product that can be used as a skin graft replacement and the process by which it is manufactured has been designed for the product to be available to the end-user off-the-shelf and for ease-of-use in practice.

The aim of this study was to develop and manufacture a dermal equivalent suitable to replace autologous skin grafts for wound closure. Several living skin equivalents (LSEs) and living dermal equivalents (LDES) have been reported previously [1-4], and although many of the currently available products were initially designed to work as skin grafts, in reality no current living product meets the rigorous requirements necessary to accomplish this function [4-6]. Rather, the dermal component of these products rapidly degrades in the wound, releasing the cells, which then contribute to wound healing by secondary intention. In contrast with earlier LSEs and LD Es that have used either a preformed collagen matrix or biodegradable synthetic mesh as the initial support system, the intent from the start of this program therefore was to develop a more robust extracellular matrix and to investigate the ability of human dermal fibroblast cells (HDFs) themsel ves to produce this matrix in vitro. It had been demonstrated previously $[4,5]$ that HDFs cast into a provisional matrix of fibrin were induced to lay down collagen, predominantly collagen I, and other extracellular matrix materials $[7,8]$. ICX-SKN skin graft replacement is based on the concept that the fibrin matrix provides a 3D fibrillar network that can be aligned by the cells in the construct and serves as a template for remodeling with the addition of collagen autosynthesized by the H DFs. In addition, the provisional matrix provides mechanical constraint that provides the stimulus for developing structure as collagen is laid down [8] and probably also provides biochemical signals to upregulate collagen synthesis [7]. 
A preliminary design specification for ICX-SKN skin graft replacement was developed which included: efficacy, convenient size and shape, ease of use, off-the-shelf availability, and permanent healing effect. In order to realize these aims, the emphasis was placed on maximizing deposition of insoluble human collagen, minimizing matrix contraction, maximizing the functional properties of the constructs (handling, rigidity/flexibility/strength, biological compatibility), and establishing a preliminary product stability profile. The studies described in this report were intended to provide a means for producing ICX-SK N skin graft replacement dermal constructs to these specifications that could be directly translated to clinical manufacture and ultimately be scaled-up both in size and number. Thus, manufacture of ICX-SKN skin graft replacement consists of four stages:

- Constructs were batch-cast by embedding HDFs into a provisional fibrin matrix and matured for 7 weeks in collagen-boosting medium to produce a first intermediate, pSK N ;

- pSK N was freeze-dried to produce the second intermediate, dSKN ;

- dSKN was sterilized by $\gamma$-irradiation to produce the third intermediate, SSK N ;

- Living HDFs were reintroduced to the SSKN matrix to yield the final product, ICX-SKN skin graft replacement, which was then packaged ready for application.

Once placed in situ, it is intended that ICXSKN skin graft replacement will maintain a biochemically balanced and moist wound environment and provide structural support for tissue regeneration and beneficial cytokines and growth factors to the wound bed. Future iterations may also be provided preseeded with keratin ocytes for the treatment of large area defects; in much of the work described here, the dermal portion alone was studied as it was felt that this was the key area requiring attention in order to provide a construct that could withstand the harsh environment of a healing wound.

We describe a series of processes through which the research concept was translated to ICX-SKN skin graft replacement constructs produced aseptically in a manufacturing facility suitable for application in Phase I clinical trials in human volunteers [9]. In this report we describe the design, development and manufacture of these constructs, and preclinical data for the use of this matrix in wound closure.
Materials \& methods

Manufacture of ICX-SKN skin

graft replacement

All products used in clinical trials were manufactured as a batch of 48 individual constructs in accordance with EU standards of good manufacturing practice (GMP) in a licensed GMP manufacturing facility at Intercytex Ltd, UK [10].

Cells \& cell growth conditions

HDFs were obtained from neonatal foreskin (donated under informed consent) essentially as described previously [11], expanded in culture to passage six at which point they were cryopreserved to produce a working cell bank (WCB). HDF cells were grown in Dulbecco's modified eagle's medium (DMEM) (Lonza, Basel, Switzerland) supplemented (D M EM -10) with $\gamma$-irradiated $10 \%$ foetal bovine serum (FBS; JRH Corp. M A, USA $)$ and L-glutamine $(0.29 \mathrm{mg} / \mathrm{ml}$; M olekula) and were used at passages 7-9 to cast into a human plasma protein, Tisseel ${ }^{\mathrm{TM}} \mathrm{VH}$ (Baxter AG, IL, USA) as described below. HDF cells sufficient to yield a batch of 48 constructs were thawed from cryopreserved stock or trypsinized from growing cultures, washed in D M EM -10 and finally resuspended in DM EM supplemented with $2 \% \mathrm{FBS}$ and L-glutamine $(0.29 \mathrm{mg} / \mathrm{ml})$ at $3 \times 10^{6} \mathrm{H} \mathrm{DFs} / \mathrm{ml}$.

\section{Production of pSKN}

The human fibrinogen sealant powder ( $T$ isseel $^{\text {Tm }} \mathrm{VH}$, Baxter AG) was reconstituted 1:1 in aprotinin solution as supplied by the manufacturer, resulting in a 'working fibrinogen sealant.' Thrombin powder (Baxter AG) was reconstituted $1: 300$ in phosphate buffered saline (PBS) $\left(-\mathrm{M} \mathrm{g}^{2+},-\left(\mathrm{a}^{2+}\right)\right.$, resulting in a 'working thrombin solution.' For each construct of ICX-SKN to be cast, working cell suspension $\left(1.8 \times 10^{6}\right.$ cells/construct), working fibrinogen sealant and working thrombin solution were mixed ( $3.7 \mathrm{ml}$ total) as indicated (see Results) and immediately dispensed into the centre of a single $(3.5 \times 2.5 \mathrm{~cm})$ well of an eight-well plastic culture plate (N unc Inc., N Y, USA). The constructs were left to gel for $30 \mathrm{~min}$ at room temperature, then were covered with DMEM-10 before being placed into a humidified incubator $\left(37^{\circ} \mathrm{C}, 5 \% \quad \mathrm{CO}_{2}\right)$ for approximately 2 days. This size of construct proved suitable for all current preclinical and clinical studies; however, larger constructs up to $10 \times 10 \mathrm{~cm}$ have been produced in other custom-modified casting chambers. 
Constructs were maintained and matured by changing the medium every 2-3 days for up to 7 weeks. In order to mature the constructs and encourage the deposition of collagen, D M EM 10 medium was replaced with total medium (TM-3:1 DMEM and Ham's-F12 [Cambrex Inc., MD, USA], $10 \% \quad F B S, \quad 0.29 \mathrm{mg} / \mathrm{ml}$ L-glutamine, $100 \mu \mathrm{M}$ ethanolamine, $6.78 \mathrm{ng} / \mathrm{ml}$ selenious acid, $50 \mathrm{ng} / \mathrm{ml}$ ascorbate, $0.2 \mu \mathrm{g} / \mathrm{ml}$ L-proline, $0.1 \mu \mathrm{g} / \mathrm{ml}$ glycine, $50 \mu \mathrm{g} / \mathrm{ml}$ gentamycin [M olekula, M O, USA], $0.4 \mu \mathrm{g} / \mathrm{ml}$ hydrocortisone [Spectrum Lab Products Inc., CA, USA], $100 \mu \mathrm{M} \quad 0$-phosphoryl-ethanolamine [M erck, $\mathrm{NJ}, U S A], 5 \mu \mathrm{g} / \mathrm{ml}$ apo-transferin, $1.35 \mathrm{ng} / \mathrm{ml}$ 3,3',5-tri-iodo-L-thyronine, $0.05 \%$ polyethylene glycol M W 3400-3700 [SigmaCorp., N J, USA], $20 \mathrm{pM} / \mathrm{ml}$ transferrin, $5 \mu \mathrm{g} / \mathrm{ml}$ insulin [Serologicals, MA, USA], $250 \mathrm{ng} / \mathrm{ml}$ amphotericin B [BufaB.V.], $62 \mathrm{ng} / \mathrm{ml} \mathrm{EGF,} 5 \mathrm{ng} / \mathrm{ml} \mathrm{TGF-} \beta$ [R $\& D$ Systems, M N , USA], 0.01 unit $/ \mathrm{ml}$ plasmin [Calbiochem, Merck KGaA, Darmstadt, Germany]) starting with the first feed $2-3$ days postcasting. M atured constructs were collected from their casting wells for freeze-drying.

\section{Production of dSKN}

Each matured pSKN construct was removed from the casting chamber and placed into a $60 \mathrm{~mm}$ Petri dish (Corning Inc., NY, USA), which was heat-sealed into a Tyvek/film pouch (Oliver Medical Products BV, Netherlands). The packaged pSKN units were placed at $-20^{\circ} \mathrm{C}$ for a minimum $12 \mathrm{~h}$ prior to freezedrying. Packaged and frozen pSKN were then placed into the vacuum chamber of a M odulyo 4K freeze-drying unit with attached RV 8 vacuum pump (Edwards) that had been precooled to $-40^{\circ} \mathrm{C}$. In order to maintain the pSKN units frozen, each frozen construct was placed onto a brass disk chilled at $-80^{\circ} \mathrm{C}$ for at least $4 \mathrm{~h}$ and the vacuum was applied. The freeze-drying cycle was conducted at $2 \times 10^{-1}$ to $8 \times 10^{-2} \mathrm{mBar}$ for a minimum of $16 \mathrm{~h}$. Each dried construct (dSKN) was then placed into a plastic roto-seal bag and was subjected to terminal sterilization.

\section{Production of SSKN}

Packaged dSK N constructs were subjected to a minimum dose of $25 \mathrm{kG} y \quad \gamma$-irradiation (Swann-M orton, Sheffield, UK). The resulting SSK N was stored excluding light at ambient temperature and constituted a raw material for the final manufacture of the ICX-SKN skin graft replacement.
Manufacture of ICX-SKN skin

graft replacement

In order to manufacture the final product, ICXSKN skin graft replacement, SSKN constructs were first removed aseptically from their packaging, visually inspected to check their integrity, rehydrated with water for injection (WFI) (JRH Inc., UK) for $10 \mathrm{~min}$ at room temperature, with two subsequent changes of water. Constructs were then washed with DMEM -10 for $10 \mathrm{~min}$. After removal of as much of the DMEM-10 as possible, HDFs $\left(0.2 \mathrm{ml}\right.$ at $9 \times 10^{6} \mathrm{cell} / \mathrm{s} / \mathrm{ml}$ in DM EM -10) were applied directly to the top surface of each construct. The constructs were placed in an incubator (humidified, $37^{\circ} \mathrm{C}, 5 \%$ $\mathrm{CO}_{2}$ ) for a minimum of $30 \mathrm{~min}$ to allow cells to adhere to the construct, at which point D M EM 10 was added to each construct and then returned to the incubator for $48 \mathrm{~h}$ prior to assessment of cell viability via reduction of Alamar Blue ${ }^{T M} \quad$ (SeroTec) and packaging for storage and/or shipping to clinic.

Constructs for cold storage or shipping were packaged into sterile polyester/poly/foil/poly pouches (Oliver Medical Products BV, $\mathrm{N}$ etherlands) containing $5 \mathrm{ml}$ of $\mathrm{CO}_{2}$-independent transport medium (L-15 medium [C ambrex] supplemented with $202.5 \mu \mathrm{g} / \mathrm{ml}$ sodium bicarbonate, $4.5 \mathrm{mg} / \mathrm{ml}$ dextrose, $24.4 \mu \mathrm{g} / \mathrm{ml}$ adenine, $0.29 \mathrm{mg} / \mathrm{ml}$ L-glutamine, $100 \mu \mathrm{M}$ ethanolamine, $100 \mu \mathrm{M} \quad 0$-phosphoryl-ethanolamine, $0.4 \mu \mathrm{g} / \mathrm{ml}$ hydrocortisone, $5 \mu \mathrm{g} / \mathrm{ml}$ human recombinant insulin, $6.78 \mathrm{ng} / \mathrm{ml}$ selenious acid, $1.35 \mathrm{ng} / \mathrm{ml}$ of 3,3',5-tri-iodo-L-thyronine, $5 \mu \mathrm{g} / \mathrm{ml}$ apo-transferrin, and $2 \% \mathrm{FBS})$. The pouches were heat sealed and visually assessed for leakage. All units were stored at $2-8^{\circ} \mathrm{C}$ during shipping and before use.

Organotypic culture of ICX-SKN

with keratinocytes

H uman keratinocytes ( $\mathrm{H} \mathrm{K} \mathrm{s}$ ) cells were obtained from neonatal foreskin (donated under informed consent), and expanded in culture. Cells were used at passage 3-4 and were expanded in EpiLife ${ }^{\circledR}$ medium supplemented with Epilife defined growth supplement (EDGS; Cascade Biologicals). HKs $\left(5 \times 10^{6}\right.$ cells/construct) were placed onto the surface of rehydrated SSK N constructs or onto the top surface (the surface al ready populated with HDFs) of ICX-SK N. HKs were made to differentiate according to established procedures [12]. Briefly, constructs were incubated at $37^{\circ} \mathrm{C}, 5 \% \mathrm{CO}_{2}$ in a humidified incubator for 2 days in epidermal 
medium (EM - 1:3mixture of DM EM (Lonza) and $\mathrm{H}$ ams-F 12 supplemented with $0.3 \% \mathrm{FBS}$, $0.29 \mathrm{mg} / \mathrm{ml} \mathrm{L}$-glutamine, $6.78 \mathrm{ng} / \mathrm{ml}$ selenious acid, $50 \mathrm{ng} / \mathrm{ml}$ ascorbate, $24.4 \mu \mathrm{g} / \mathrm{ml}$ adenine, $50 \mu \mathrm{g} / \mathrm{ml}$ gentamycin, $100 \mu \mathrm{M}$ ethanolamine, $0.4 \mu \mathrm{g} / \mathrm{ml}$ hydrocortisone [Spectrum Lab. Products Inc], $100 \mu \mathrm{M} 0$-phosphoryl-ethanolamine, $1.35 \mathrm{ng} / \mathrm{ml}$ of $3,3^{\prime}, 5$-tri-iodo-L-thyronine, $5 \mu \mathrm{g} / \mathrm{ml}$ insulin, $5 \mu \mathrm{g} / \mathrm{ml}$ apo-transferrin, $10 \mathrm{ng} / \mathrm{ml} \mathrm{EGF}$ and $0.628 \mathrm{ng} / \mathrm{ml}$ progesterone [Amersham]). After 2 days this medium was replaced with extracellular matrix containing $265 \mu \mathrm{g} / \mathrm{ml} \mathrm{CaCl}_{2}$ (Sigma Corp.), but omitting $\mathrm{EGF}$ and constructs were incubated for a further 2 days. To allow the stratification of the epithelial layer the constructs were aseptically transferred onto the polycarbonate membrane of transwell dishes (Corning Inc.) and maintained in medium omitting both progesterone and $\mathrm{CaCl}_{2}$, achieving a fluid level just to the surface of the carrier membrane to maintain a liquid-air interface. Constructs were maintained in a humidified incubator at $37^{\circ} \mathrm{C}, 5 \% \mathrm{CO}_{2}$ for 7 days with changes of the media every 2-3 days. Finally, constructs were maintained in medium omitting ascorbate for a further 10 days with medium changes every 2-3 days.

\section{Analyses of ICX-SKN skin \\ graft substitute \\ Histology}

Samples for histological analysis were prepared by embedding in paraffin wax and sections $(5 \mu \mathrm{m})$ were cut using a rotary microtome (Leica, IL, USA), placed onto microscope slides, then de-waxed and stained with hematoxylin and eosin $(H \& E)$ stain. Following staining slides were mounted in DPX mounting media (BDH, UK) and coverslips applied. Digital images were captured using a Nikon 4500 CoolPix digital camera under light microscopy.

\section{Immunocytochemistry}

Samples were embedded in optimal cutting temperature (OCT) embedding medium (RA Lamb, UK) and sections ( $10 \mu \mathrm{m})$ were obtained using rotary microtome (Leica). Sections were thawed at room temperature for $10 \mathrm{~min}$, fixed in icecold acetone for $5 \mathrm{~min}$ and air-dried. Air-dried sections were rinsed in three changes of a wash buffer containing $1 \%$ normal goat serum (N GS) and $0.1 \%$ TritonX100 in PBS for $10 \mathrm{~min}$. Sections were blocked for $30 \mathrm{~min}$ at room temperature using $20 \%$ NGS blocking solution. The slides were drained to remove the blocking solution and the sections were incubated for $1 \mathrm{~h}$ at room temperature in the primary antibody. Primary antibodies (AbC am, UK) were: antihuman collagen 1 (1:200), antihuman fibrin (1:100), antihuman laminin-5 (1:250), antihuman keratin (1:250), and antihuman involucrin (1:200). Sections were rinsed in three changes of PBS for 10 min each, and incubated for a minimum $60 \mathrm{~min}$ at room temperature in the appropriate fluorescein isothiocyanate (FITC)conjugated secondary antibody (Jackson I mmunoresearch) at 1:200 dilution. Finally, sections were rinsed in three changes of PBS for $10 \mathrm{~min}$ each, mounted in diamidinophenylindole (D API)-containing mounting solution (Vectashield, CA, USA) and coverslipped. Images were captured using a Nikon Coolpix 4500 digital camera mounted onto a fluorescence microscope (Leica) using an appropriate fluorescence filter.

Phalloidin staining of ICX-SKN constructs Repopulated constructs were washed in citrate buffer solution (CBS) buffer (10 mM 2-(N -morpholino)ethanesulfonic acid (MES) pH 6.1; $138 \mathrm{mM} \mathrm{KCl} ; 3 \mathrm{mM} \mathrm{M} \mathrm{gCl2;} 2 \mathrm{mM}$ ethylene glycol tetraacetic acid (EGTA); $11 \%$ sucrose), then fixed in $4 \%$ paraformaldehyde in CBS buffer for $1 \mathrm{~h}$ at $2-8^{\circ} \mathrm{C}$. Samples were then frozen in OCT embedding medium and $8 \mu \mathrm{m}$ sections were cut and placed onto microscope slides. Samples were then rinsed in TBS buffer (0.02 M Tris (pH 7.4-7.5); $0.15 \mathrm{M} \mathrm{NaCl}$ ), permeabilized in TBS $0.5 \%$ TritonX 100 for $10 \mathrm{~min}$ and rinsed three times in TBS $0.1 \%$ TritonX 100 . Sections were pretreated in block buffer (TBS $0.1 \%$ TritonX100, 2\% BSA) for $10 \mathrm{~min}$ then incubated with fluorescence-phalloidin $(10 \mu \mathrm{g} / \mathrm{ml}$ in block buffer) for $20 \mathrm{~min}$. Samples were rinsed again in TBS $0.1 \%$ TritonX100, drained well, mounted with D APIcontaining mounting solution (Vectasheild) and viewed directly by fluorescence microscopy. Images were stored as electronic files.

\section{Live cell labeling with Cell Tracker ${ }^{\mathrm{TM}}$ \\ CM-Dil dye}

HD Fs used for repopulation of SSK N constructs were stained with Vybrant CM -Dil (Invitrogen, CA, USA) dye according to the manufacturer's instructions. Briefly, cells were collected from growing cultures or were recovered from cryopreservation and washed twice in DMEM and then resuspended in DMEM (10 ml). CM-Dil dye $(10 \mu \mathrm{l}$ of $10 \mathrm{mM}$ in Dimethyl sulfoxide [D M SO ; Sigma Corp.]) was added to the cell 
suspension and the cells were incubated at $37^{\circ} \mathrm{C}$ for $30 \mathrm{~min}$. The cell suspension was then pelleted by centrifugation ( $5 \mathrm{~min}$ at $500 \mathrm{~g}$ ) and cells were washed twice in DM EM - 10 to remove all traces of the dye. Cells were finally resuspended in DMEM-10 $\left(9 \times 10^{6} \mathrm{cell} / \mathrm{s} / \mathrm{ml}\right)$ and were applied to the rehydrated SSKN matrix as described previously.

\section{Differential scanning calorimetry}

Differential scanning calorimetry (DSC) analyses were performed on D SCQ 100 machine (TA Instruments, DE, USA). Briefly, a sample (5 mg) collected on day 0 postcasting or fully matured SSKN, or as a control, porcine dermis, was placed into the aluminum pan (TA Instruments). Prior to analysis samples were fully rehydrated with a drop of PBS (Sigma Corp.) for at least $5 \mathrm{~min}$ at room temperature. Pans were hermetically sealed and inserted into the instrument. The samples were heated from $0-120^{\circ} \mathrm{C}$ at $5^{\circ} \mathrm{C} / \mathrm{min}$. The results were analyzed using $T A$ Instruments software and denaturation temperature ( $\mathrm{Td}$ ) of the major component of the matrix was established. Porcine dermis, used as a control, was prepared by removal of the epidermis using dispase solution (Sigma Corp.) for $12 \mathrm{~h}$ at $4^{\circ} \mathrm{C}$. The epidermis was scraped from the dermis, the dermis washed several times in PBS, and finally freeze-dried and sterilized.

Scanning electron microscopy.

Scanning electron microscopy (SEM) was performed by the electron microscopy unit at $M$ anchester U niversity, Faculty of Life Sciences using a SEM (Cambridge Instruments, UK). The freeze-dried (dSKN) samples were broken into small pieces using tweezers and were attached onto double-sided adhesive tape and placed on the top of carbon coated tabs. The samples were then coated with gold using argon gas to disperse the gold molecules onto the samples. Samples were then inserted into the chamber of the SEM and the chamber evacuated to obtain vacuum for a minimum of $30 \mathrm{~min}$. Images were then obtained using scanning microscopy image software (Cambridge Instruments, UK).

\section{Resistance test}

Repopulated constructs were tested to breaking point using a M ultitest force gauge (M ecmesin) equipped with a $10 \mathrm{~N}$ ewton load cell. The constructs were gripped with clamps attached to the force gauge in such a way that the force was applied along the longer side of the construct.
The constructs were first 'exercised' at $2 \mathrm{~mm} / \mathrm{min}$ three times to a displacement of $1 \mathrm{~mm}$ each cycle, and allowed to relax to their original position between displacements, then finally displaced to breaking point.

Determination of cell viability

Alamar Blue $^{\mathrm{TM}}$ (Serotec, NC, USA) assay for metabolic activity of cells was essentially performed according to manufacturers' instructions on ICX-SKN constructs. Briefly, a 10\% Alamar Blue $^{\mathrm{TM}}$ solution was prepared in $\mathrm{CO}_{2}$-independent transport medium. The construct was placed into a container to which the $10 \%$ Alamar Blue ${ }^{T M}$ solution $(6 \mathrm{ml})$ was added, then placed at $37^{\circ} \mathrm{C}$. Alamar Blue ${ }^{T M}$ reduction was measured in duplicate samples at 30-min intervals at 570 and $600 \mathrm{~nm}$ wavelengths. The reduction of Alamar Blue was calculated according to manufacturers' instructions.

\section{Open wound assay}

Full-thickness excisions $\left(0.8 \times 1.8 \mathrm{~cm}^{2}\right)$ were made to the dorsal skin of anaesthetized male CD-1 nu/nu mice (Charles River Laboratory, MA, USA). A piece of rehydrated SSKN or ICX-SK N skin graft replacement, cut to match the size and shape of the excision, was placed onto the wound. Four sutures were made on the four corners to fix the graft in place and a piece of adaptic nonadhering dressing gauze (Johnson and Johnson) was placed onto the graft before the wound was bandaged. Wounds were observed at 7-day intervals and the graft tissue was harvested from sacrificed animals for histological analysis

\section{Results}

\section{Development of pSKN matrix}

Once placed in situ, it is intended that ICX-SKN skin graft replacement will maintain a biochemically balanced and moist wound environment and provide robust structural support for tissue regeneration as well as beneficial cytokines and growth factors to the wound bed. D uring manufacture of pSKN matrix, fibroblasts initially embedded in fibrin in vitro begin the process of remodeling the provisional matrix by destruction of fibrin whilst incorporating insoluble collagen into the matrix [7]. A presumed threshold exists above which the two processes occur at a rate which means that fibroblasts are continually embedded in a dynamic, insoluble matrix composed variably of fibrin and/or collagen [9]. Below this threshold, destruction of fibrin runs 
ahead of incorporation of collagen and the fibroblasts are released into the liquid medium. In order to embed HDF cells in a stable 3D scaffold variously composed of human fibrin and/or human collagen, different concentrations of fibrinogen and thrombin were tested. The aim was to obtain constructs that were easy and reliable to cast and that did not dissolve or contract during 7 weeks of maturation in collagen-boosting medium (TM). Several formulations were tested, including combinations of the precursor to human fibrin, fibrinogen, at initial concentrations of $7 \mathrm{mg}$ and $1 \mathrm{mg}$ and human thrombin at 83 or 8.3 units, respectively, per construct (Table1). In order to slow the process of cleavage of fibrinogen by thrombin to produce fibrin, and allow sufficient time for casting the mixture, all reagents were chilled to $2-8^{\circ} \mathrm{C}$. Each construct was cast individually into a $3.5 \times 2.5 \mathrm{~cm}$ casting chamber, taking care that the surface tension of the casting mix was maintained once cast.

It was difficult to effectively cast constructs using formulations of high thrombin (Formulations 1 and 3, Table1) as the fibrinogen/cell mix tended to set immediately on contact with thrombin in the casting pipette. Those that could be cast resulted in uneven constructs or constructs that contracted during maturation (Figre1A). C onversely, constructs cast with high fibrinogen and low thrombin (Formulation 2, Table1) dissolved before sufficient collagen was incorporated (data not shown). The most consistent results were obtained using low fibrinogen and low thrombin (Formulation 4, Table1) and the precooled casting mix allowed a reasonable 'casting window' of 30-60 s, which resulted in even and well-formed constructs (Figre 1B). In addition, after maturation in TM ,

\section{Table 1. Formulations for PSKN.}

\begin{tabular}{lrrr} 
Formulation & $\begin{array}{r}\text { Fibrinogen } \\
\mathbf{m g} \text { /construct }\end{array}$ & $\begin{array}{r}\text { Thrombin } \\
\text { units/construct }\end{array}$ & $\begin{array}{r}\text { Final } \\
\text { volume }(\boldsymbol{\mu l})\end{array}$ \\
$\begin{array}{l}\text { 1. High } \\
\text { fibrinogen/high } \\
\text { thrombin }\end{array}$ & 7 & 83 & 3670 \\
$\begin{array}{l}\text { 2. High } \\
\text { fibrinogen/low } \\
\text { thrombin }\end{array}$ & 7 & 8.3 & 3670 \\
$\begin{array}{l}\text { 3. Low } \\
\text { fibrinogen/high } \\
\text { thrombin }\end{array}$ & 1 & & \\
$\begin{array}{l}\text { 4. Low } \\
\text { fibrinogen/low } \\
\text { thrombin }\end{array}$ & & 83 & 3700 \\
\hline
\end{tabular}

the constructs obtained from the latter formulation had not contracted or dissolved and were easily excised from the casting dishes (Figre1C) without being damaged.

In addition to entrapment of HDFs into a provisional matrix, the development of a prototype suitable for use as a dermal substitute in clinical applications required that H D Fs synthesize and deposit substantial amounts of collagen and other components of extracellular matrix into robust insoluble structures in order to resist the physicochemical forces at work on human skin. It has been shown that supplementing media with factors such as TGF- $\beta$, insulin, ascorbate and plasmin can induce collagen deposition $[7,8]$. We developed TM by combining components already described $[7,8,201]$, in addition to other cell-sustaining components. In order to evaluate the effect on collagen and ECM components synthesis by H D Fs, constructs (formulated with low fibrinogen and thrombin as described above) were subjected to threetimes per week feeding [8] with TM or with TM omitting EGF, TGF- $\beta$, insulin and plasmin (MPM) and sample constructs were collected at 7, 14, 21 and 52 days of maturation. Constructs fed in TM medium over the period of maturation became increasingly opaque relative to those constructs fed with M PM (Figre2A), suggesting that ECM proteins were being laid down in the constructs during maturation. By contrast, constructs maintained in M PM retained a transparent, gel-like appearance. In addition, compared with M PM , constructs fed with TM were more robust in their handling properties; whereas MPM-fed constructs were easily torn upon removal from the casting dish, TM constructs remained intact upon manipulation.

TM - and MPM-treated constructs were immunostained using antihuman-collagen I antibody (Figure2B) and revealed that collagen I was present in constructs maintained in TM to a greater extent than in constructs maintained in M PM . Furthermore, collagen I was deposited as early as 10-14 days of maturation (Figre2B iv) in those constructs maintained in TM .

Comparative analysis by DSC of constructs matured in TM (pSKN), porcine dermis and constructs composed largely of fibrin (i.e., day 0 (d0) of maturation) was performed (Table2). O ne major component from each matrix was detected; however, the $\mathrm{T} d$ of these major components differed substantially between the two constructs, showing that during maturation the initial fibrin matrix was converted. 
Figure 1. Constructs cast with different formulations of fibrinogen and thrombin.

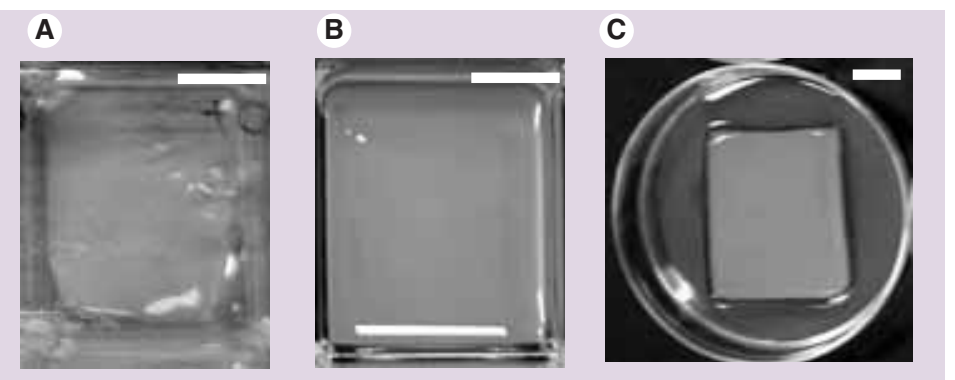

(A) High fibrinogen and thrombin (initial $7 \mathrm{mg}$ and 83 units per construct, respectively) (B) Low fibrin and thrombin (initial $1 \mathrm{mg}$ and 8.3 units per construct, respectively) (C) Construct as in B at 49 days maturation after removal from casting dish. Scale bar: $10 \mathrm{~mm}$.
Demonstrated by comparison with the $\mathrm{Td}$ of porcine dermis, the fibrin matrix was converted to collagen during the 49-day maturation period.

Development of dSKN, SSKN \& ICX-SKN skin graft replacement

pSK $\mathrm{N}$ was produced in 7 weeks by intensive feeding with medium supplemented with a cohort of growth factors designed to boost the synthesis and deposition of collagen. At this point, pSKN could be used directly in clinical application. H owever, the ability to preserve the matrix at this stage would add value to pSK $\mathrm{N}$ by both allowing for an increased shelf-life of the product and also enabling material to be stockpiled for use. One approach for increasing shelf-life of similar products has been cryopreservation [101]. H owever, the need for specialized storage both at manufacture site and clinic, and the requirement for complex recovery methods from cryopreservation before application rendered this method of preservation for PSKN unsuitable in the manufacture of ICX-SKN skin graft replacement.

Freeze-drying was chosen as the method for preservation of the matrix since it could provide a means of storing the PSK $N$ product (known as dSK $N$ once dried) at ambient temperature for long periods. pSK N constructs were subjected to washing in different solutions prior to freezedrying with the aim of obtaining constructs that maintained their 3D structure and rectangular shape, without becoming brittle. In addition, since the process of freeze-drying destroyed cells within the pSKN construct, the freezedried constructs had to maintain the ability to sustain cells once repopulated with fresh HDFs (i.e., remain 'cell friendly'). Constructs were freeze dried in Soltran, $0.1 \mathrm{M}$ Tris pH 7.0 and TM medium and were subsequently rehydrated and repopulated with fresh HDFs (see M aterials and Methods) then assessed for viability of the fresh HDFs.

Constructs dried in osmotically balanced biological sample preservation solution (Soltran), lost integrity upon freeze-drying (Figure3A). H owever, pSKN matrices freeze-dried in Tris or TM remained intact, maintaining their thickness and flexibility even when dry (Figre3A iv). These constructs were readily rehydrated and, in contrast to constructs freeze-dried in other formulations (data not shown), both were able to support attachment and sustained cell viability once repopulated with fresh HDFs (Figure3B). D espite comparable results freeze-drying constructs in Tris, freeze-drying directly from TM was chosen since it removed the need for a further manipulation (i.e., washing pSKN constructs in Tris), an important consideration when designing a manufacturing process for cell-therapies.

To view the 3D structure of freezedried constructs in more detail, constructs were analyzed by SEM. Constructs freeze-dried from TM media showed a relatively well-preserved 3D structure of the collagen matrix, with evident pores of variable size (Figure 3 C).

The dSK N matrix was sterilized by $\gamma$-irradiation (resulting in SSKN ) to render it suitable for final product assembly and application in clinical trials. $\gamma$-irradiation with a terminal minimal dose of $25 \mathrm{kG}$ y is an approved method of sterilization of medical products and devices [13]. It is also a nonproduct-contact sterilization method that does not leave residues on the product or product packaging. It has been reported previously that $\gamma$-irradiation has a detrimental effect on integrity of collagen matrix [14,15]. Sample pSKN, dSKN and SSKN constructs were subjected to cyclic stress and the breaking stress determined. In contrast to pSKN, whose strength was not measurable with the test method utilized (data not shown), rehydrated and repopulated dSKN and SSKN constructs demonstrated resistance to cyclic stress (Figre4A) and had comparable ultimate tensile strength (Table3), indicating the matrix was not compromised by the applied dose of $\gamma$-irradiation. Furthermore, SSKN had a demonstrable capacity to sustain viable cells on the matrix once rehydrated and repopulated as described above (Figre4B) to yield the final product, ICX-SKN skin graft replacement. 


\section{Figure 2. Effect of feeding regime on pSKN constructs.}

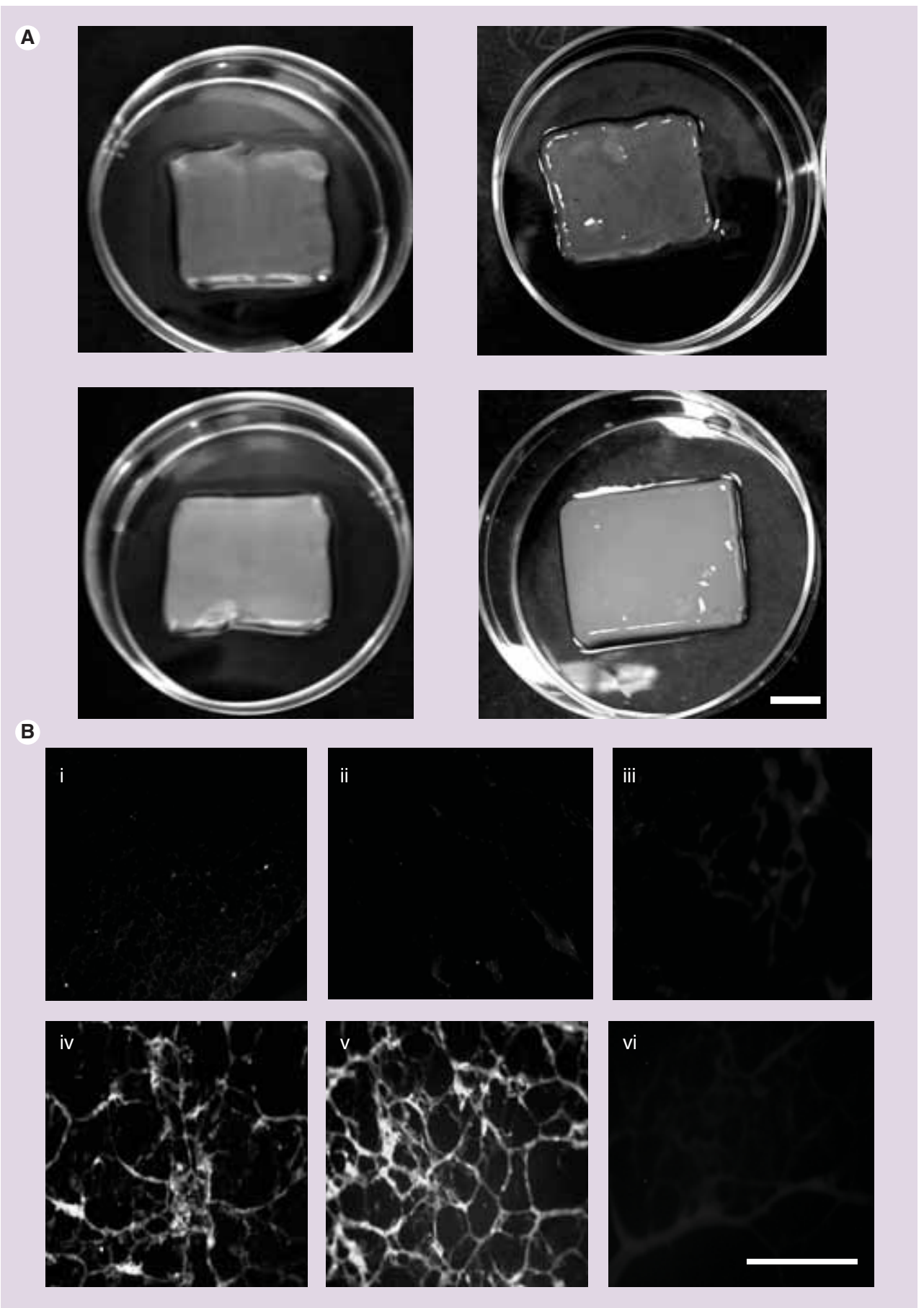

(A) Gross appearance of constructs fed three times/week for 7 (left panels) or 52 (right panels) days, as indicated, with M PM (top row) or TM (bottom row). Scale bar: $10 \mathrm{~mm}$. (B) Collagen I in constructs (i) immediately after casting or fed with MPM for (ii) 14 days or (iii) 52 days or fed with TM for (iv) 14 days or (v) 52 days. Control (vi) is construct fed with TM for 52 days but eliminating the collagen I antibody. Scale bar: $250 \mu \mathrm{m}$.

Stress fibers are maintained in living cells and staining with fluorescent-phalloidin allowed viewing of the live cells on or within the matrix
(Figre4C) without interference from cells that had been within the construct at the time of freeze-drying (i.e., dead cells). That the matrix 
Table 2. Comparative differential scanning calorimetry analysis.

\begin{tabular}{lr}
\hline Sample & Td (mean \pm SD) \\
\hline Fibrin construct (d0) & $55.6 \pm 3.0$ \\
\hline pSKN (d49) & $43.4 \pm 1.3$ \\
Porcine dermis & $46.4 \pm 1.6$ \\
\hline
\end{tabular}

SD: Standard deviation; Td: Denaturation temperature.

was able to support attachment of living H D Fs was supported by detection on repopulated ICX-SKN constructs of actin-stress fibers 2 days after reintroduction of live $H$ DFs, which were present mostly on the top surface of the construct to which they were applied. The nuclei of cells present when pSKN was freeze dried were also evident.

When stored at $2-8^{\circ} \mathrm{C}$ in $\mathrm{CO}_{2}$-independent transport media (see $M$ aterials and $M$ ethods), ICX-SKN skin graft replacement was stable for at least 5 days (Figure4B) maintaining approximately $75 \%$ of initial cellular activity.

Addition of keratinocytes to ICX-SKN skin graft replacement

ICX-SKN skin graft replacement is a platform that has the potential to be developed as a fully differentiated bilayered skin substitute. In order to investigate the potential of constructs to support attachment and differentiation of keratinocytes, human neonatal keratinocytes were seeded onto ICX-SK N skin graft replacement constructs and onto rehydrated SSKN constructs. Keratinocytes were incubated on the constructs for 2 days before being exposed to EM for a further 2 days and finally brought to the air-liquid interface to induce keratinocyte differentiation and cornification (see $M$ aterials $\&$ methods). Constructs repopulated with HDFs (ICX-SKN) and/or HKs (SSKN) both expressed keratin (Figure5A). Involucrin, a specific marker of keratinocyte differentiation, was detected on ICX-SKN constructs after exposure to differentiation induction but was not detected in any constructs before differentiation induction. A low level of involucrin expression was observed on rehydrated SSK N constructs exposed to differentiation conditions onto which only keratinocytes had been seeded. ICX-SKN constructs (previously populated with HDFs) onto which keratinocytes were seeded displayed clear evidence of involucrin expression, stratification and cornification (Figre5B). C onstructs were examined for expression of laminin-5, a basement membrane marker expressed by $\mathrm{HKS}$ in the presence of HDFs; only ICX-SKN constructs repopulated with $\mathrm{H} \mathrm{Ks}$ and $\mathrm{H}$ D Fs demonstrated robust laminin-5 expression but stratification was observed in both types of construct (Figre5C).

Preclinical study of ICX-SKN skin graft replacement

ICX-SKN skin graft replacement and rehydrated SSKN constructs were grafted onto full thickness excisions made on the dorsal surface in male nu/nu mice. In total eight of each graft type were applied and no adverse effects of the grafts to animals were observed over 28 days. All animals recovered well and integration of the grafts was noted in all cases (Figre6A) after application of both graft types, and all wounds were completely healed by 28 days. Epidermis formed over the graft site. Although there was extensive contraction to the wound sites, no scarring or blistering was observed. It was felt that contraction was due to dehydration of the grafts during the remodeling period, resulting from the difficulty of maintaining hydration in the animal model. Care was taken during the clinical study on human volunteers to ensure that secondary dressings maintained graft hydration and wound contraction was not observed [9].

At each time-point, two animals from each experimental group were sacrificed and the graft and surrounding tissue was excised and analyzed by $\mathrm{H} \& \mathrm{E}$ tissue stain. ICX-SKN skin graft replacement constructs appeared to induce accelerated healing relative to rehydrated SSK N matrix as samples taken at 7 days postgrafting revealed that, whereas host epithelium had begun to migrate over the ICX-SKN skin graft replacement, there was no evidence of migration over the SSKN graft from the same time-point (Figre6B, compare top right to top left). H owever, both types of graft were completely covered by epidermis after 28 days (Figure6B bottom panels). In addition, the newly formed epidermis appeared to be bonded well with underlying ICX-SKN skin graft replacement matrix, without any gaps or blistering, suggesting that proper attachment of both layers was achieved.

The expression of laminin-5 and collagen-IV, both protein markers of basement membrane, was investigated in ICX-SKN grafts excised at day 28. In order to distinguish expression of these proteins in basement membranes present in the host tissue and expression over the grafted 


\section{Figure 3. Effect of freeze-drying solutions on dSKN constructs.}

A

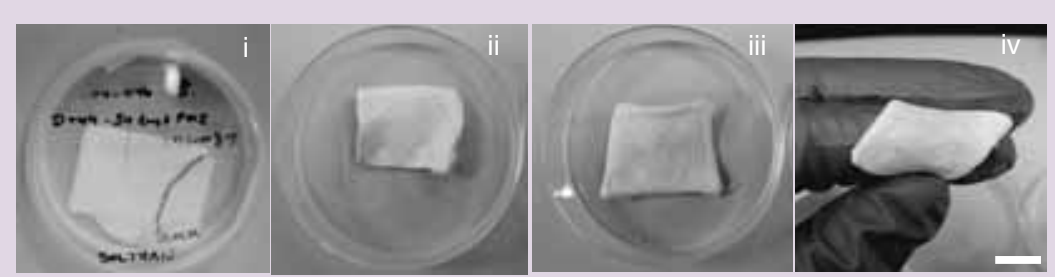

B

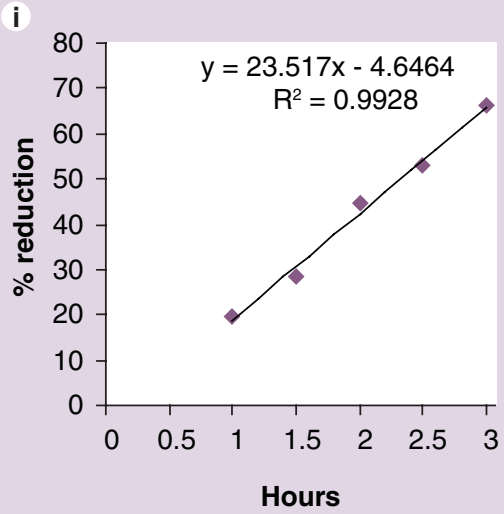

ii

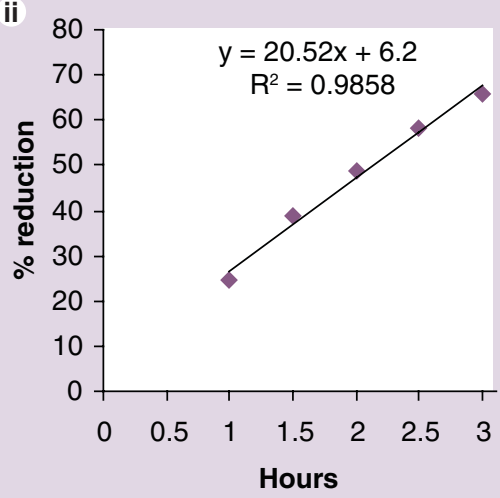

C

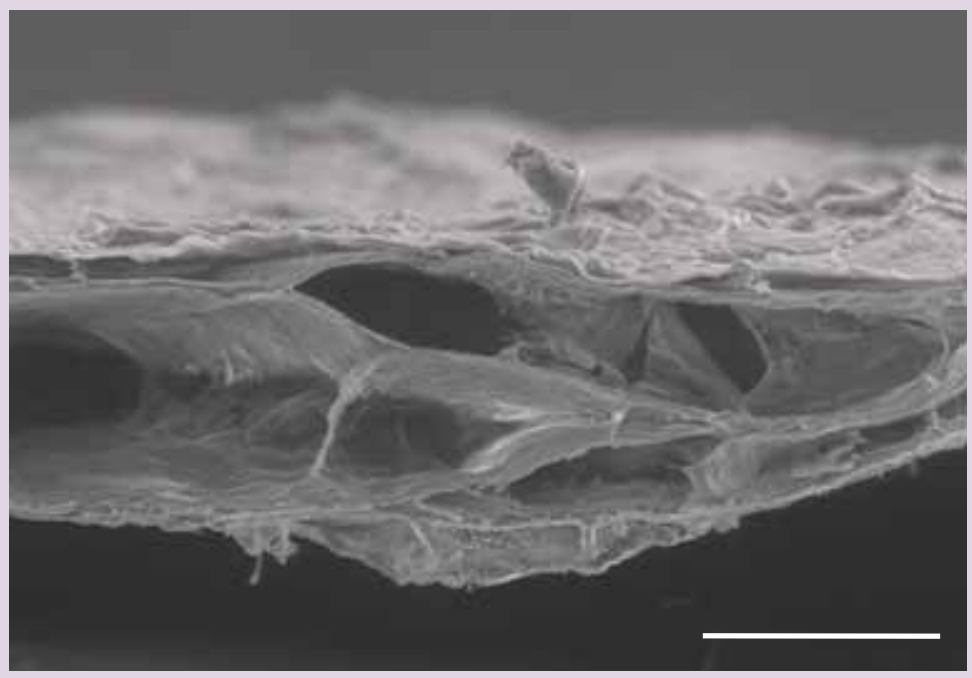

(A) Gross appearance of constructs freeze-dried in (i) Soltran, (ii) 0.1 M Tris pH 7.0, (iii, iv) TM. Scale bar: $10 \mathrm{~mm}$. (B) Example of metabolic activity of cells repopulating dSKN freeze-dried as in (i) $0.1 \mathrm{M}$ Tris pH 7.0 $(n=9)$, (ii) TM ( $n=51)$ as measured by reduction of Alamar Blue ${ }^{T M}$ (\% reduction/h) (C) Scanning electron micrograph of dSKN construct freeze-dried in TM. Scale bar: $100 \mu \mathrm{m}$.

TM: Collagen-boosting medium.

material, HDFs used to repopulate ICX-SK N constructs were first labeled with Vybrant CM-Dil dye. Collagen-IV and laminin-5 were present in the membrane directly overlying the grafts at construct-host epithelia boundary (Figre6C) and staining for these proteins was contiguous between ICX-SKN skin graft replacement matrix and host epithelium. In addition, HDFs were detected at 28 days after graft application, suggesting persistence of applied cells in the healed wound (Figre6 $\mathrm{C}$ iii). $\mathrm{H}$ airs or hair follicles around the graft on one of the subject animals (Figure6A \& C middle panel) were observed. H owever, their presence was most likely owing to variability in phenotypic expression in this strain and not as a result of the graft. 


\section{Figure 4. Characterization of ICX-SKN skin graft replacement.}

B i

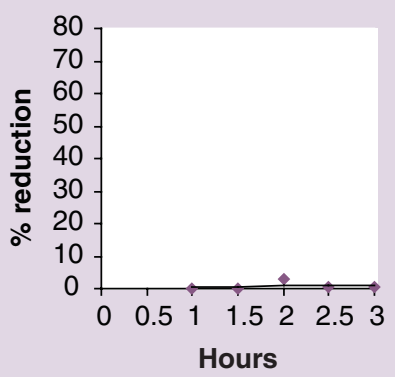

ii

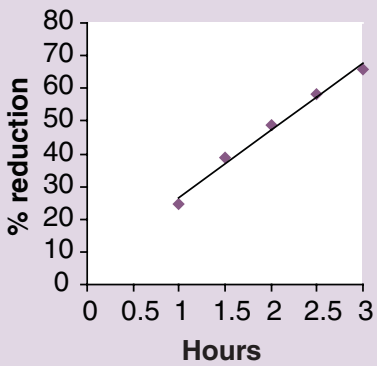

iii

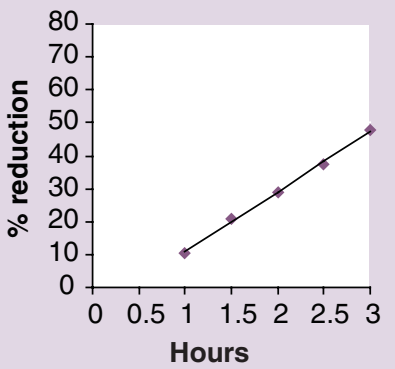

C
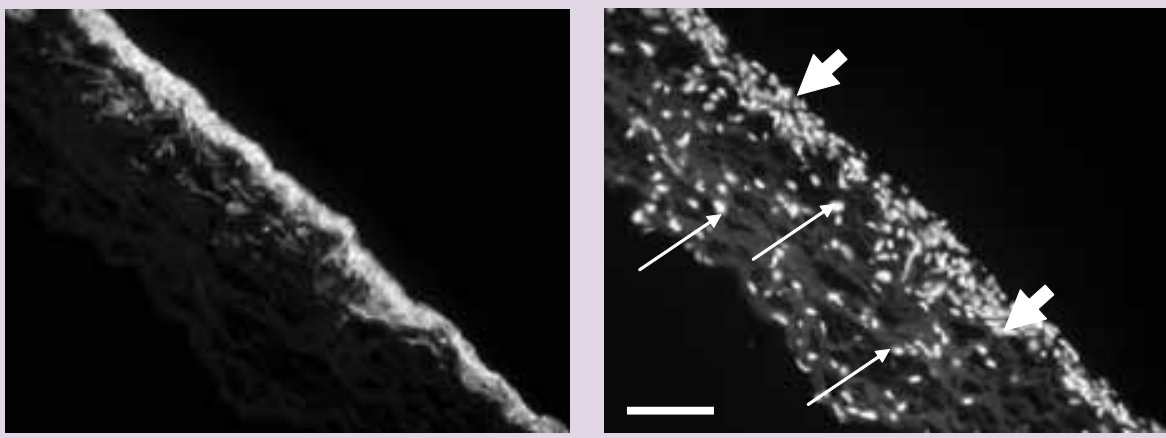

(A) Stretch and strength characteristics of an example (freeze-dried and sterilized) SSKN constructs (N/seconds) on day 0 post repopulation with HDFs $(n=51)$. (B) M etabolic activity of example (i) dSKN $(n=2)$ and ICX-SKN constructs (ii) 0 days $(n=51)$ and (iii) 5 days $(n=18)$ in cold storage as measured by reduction of Alamar Blue ${ }^{T M}$ (\% reduction/hour) (C) Stress fibers in fresh HDFs (left) stained with phalloidin and nuclei (right) from fresh HDFs (large arrows) and original HDFs (small arrows) stained with DAPI in a cross-section of ICX-SKN skin graft replacement. Scale bar: $250 \mu \mathrm{m}$.

DAPI: Diamidinophenylindole; HDF: Human dermal fibroblast cells.

\section{Discussion}

ICX-SKN skin graft replacement can be regarded as a platform product: a matrix consisting largely of human collagen autosynthesized by H D F s over a 7-week maturation period. In order to maximize the utility of this matrix, it is freeze-dried and sterilized so that it can be stored under ambient conditions and can be regarded, essentially, as a raw material in the manufacturing process of the final product. The platform can be repopulated with living cells and used in clinical applications, one of which we have already described [9]. 


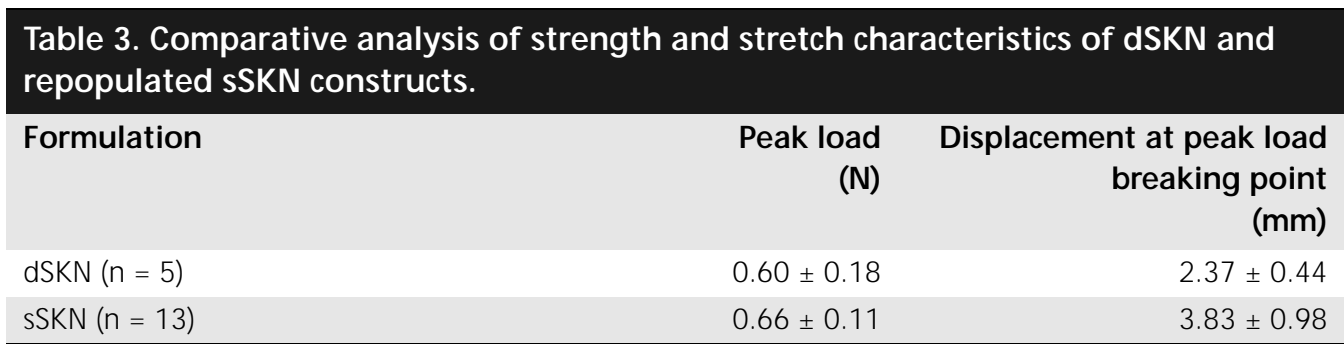

Datashown arethemean \pm standard deviation.

A number of consultant surgeons in dermatology, plastic and reconstructive, and burns surgery were contacted in order to garner their expert opinion in concrete terms on the desirable characteristics of a skin graft replacement. Among the key desirable characteristics for such a product was persistent healing effect such that, although over time physiologic processes should remodel the application, the wound would be permanently closed. In addition, efficacy, easeof-use and off-the-shelf availability were felt to be essential for the success of such an application. From the original research concept the product was designed and developed with this utility in mind.

Although many of the currently available cell therapy-based products were initially designed to work as skin grafts, in reality no current product meets the rigorous requirements necessary to accomplish this function $[16,17]$. Unlike some of these earlier products that were based on cells embedded in purified bovine collagen [17], ICX-SKN skin graft replacement is based on synthesis of collagen by HDFs embedded in a provisional matrix of human fibrin over a period of maturation. Earlier work by Tuan, Neidert and others demonstrated that in similar compositions, HDFs in bovine fibrin were induced to lay down collagen and other extracellular matrix materials $[7,8]$. As the construct matured, the original fibrin was augmented or replaced by collagen that became, or could be induced to become, cross-linked. We would distinguish ICX-SKN on the basis that the collagen produced by allogeneic H D Fs embedded initially in the human fibrin gel is autosynthesized and thus more likely to resemble collagen laid down in the authentic wound-healing process. UItimately, the extracellular matrix manufactured by HD Fs as the construct matures lends the functional properties of tensile strength, elasticity and flexibility to the ICX-SK N constructs.

In order to maximize the clinical and commercial potential of the extensive maturation period, the manufacturing process that results in the final product, ICX-SKN skin graft replacement, encompasses several processes in a discontinuous manufacturing cycle (Figre7). The construct is cast and matured to produce pSKN , the first intermediate. The 7-week aseptic process during which HDFs were stimulated to lay down collagen and other extracellular matrix proteins posed several logistical hurdles:

- Extensive manipulation (feeding, etc.) exposed the nascent constructs to the possibility of contamination, so antibiotics were included in the feeding medium

- Since PSKN was produced by living cells the shelf-life would be limited if pSK $N$ were the final product.

Thus, a process was designed in which $\mathrm{pSKN}$ was instead an intermediate in manufacture of ICX-SKN, whose final manufacture can be scheduled according to customer demand. The second intermediate, dSKN, is the result of freeze drying pSK $N$, allowing preservation of the matured matrix which could, upon $\gamma$-irradiation, be stored at ambient temperature as a raw material. Irradiation, to produce the third intermediate, SSKN, was made necessary by the presence in the pSKN manufacturing process of antibiotic, which is bacteriostatic rather than bacteriocidal, and together with aseptic processing in a qualified clean-room environment, ensured sterility of the entire batch. Together the processes of freeze-drying and irradiation consume 1 week. Finally, SSKN was rehydrated, washed and repopulated with fresh HDFs to produce ICX-SKN skin graft replacement, which was packaged and shipped to the clinic. Relative to the manufacture of pSKN, ICX-SKN is ready for shipment after 2 days of repopulation. The capacity to hold SSKN under ambient conditions together with accessibility to a fully qualified bank of H D Fs results in product that can be quickly assembled when needed and can be available off-the-shelf to the end user. In addition, at several points, processes were rationalized to reduce manipulation of the product, for 
Figure 5. Addition of keratinocytes to ICX-SKN skin graft replacement.

A
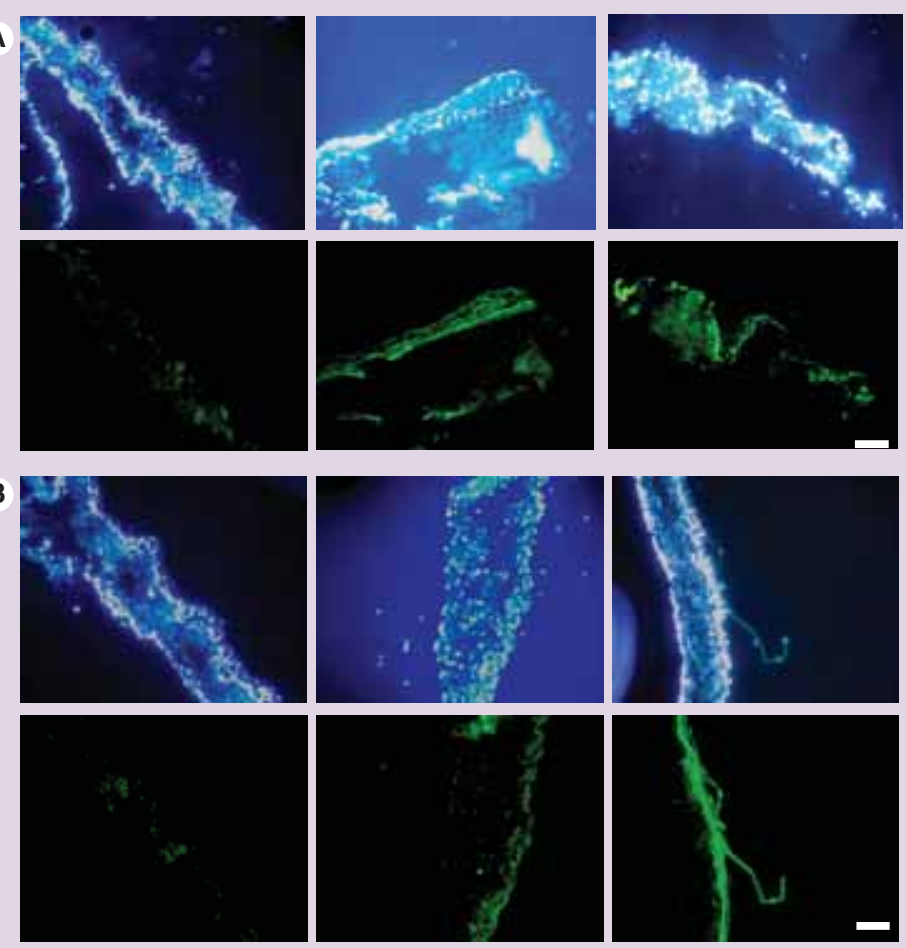

C
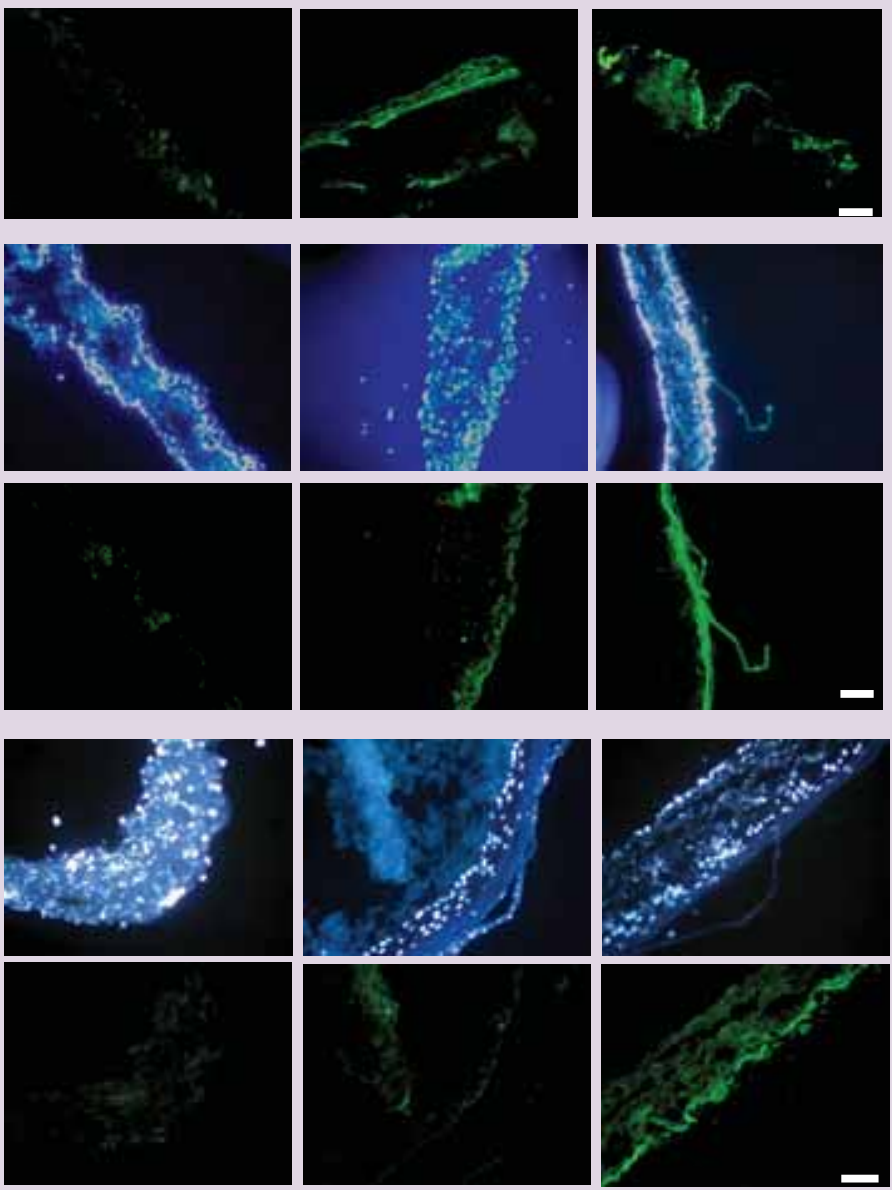

Rehydrated SSKN (middle panels) or ICX-SKN (right-hand panels) constructs were populated with human keratinocytes and exposed to differentiation conditions in vitro. Sections were stained for (A) keratin, (B) involucrin and (C) laminin-5 (bottom panels) and diamidinophenylindole (top panels). Control sections (left hand panels) were stained with secondary antibody only. Scale bar: $250 \mu \mathrm{m}$.

example, the choice of freeze-drying medium, $T M$, which requires no washing of the pSKN constructs prior to freeze drying. The conditions of packaging and shipping mean that the product can be applied directly from the package with no further preparation by the end-user.

We have demonstrated here in preclinical models and in first-in-man use [9] that the platform product is biologically acceptable and integrates well with host tissues and that host epidermis readily colonized grafts in both cases.
Contraction in the animal model was observed and contrasts with lack of contraction observed in the Phase I clinical trial [9]; although the relative size of wound created in the preclinical study was larger and hydration of wounds in animal models is technically difficult, perhaps this outcome also underlines the paucity of adequate animal models for the study of wound healing. The development of epidermis over the graft was apparently accelerated by the application of keratinocytes and HDFs to the graft, although grafts repopulated with keratinocytes al one eventually developed epidermis as well. In addition, we have demonstrated that keratinocytes applied in vitro colonize the platform and that specific protein markers of basement membranes, collagen IV and laminin-5, were expressed. Furthermore, expression of both proteins was observed at the site of graft in preclinical models. Although delamination was not observed in the mouse wound treated with ICX-SKN matrix at 28 days, delamination upon histological processing was evident in some of the clinical subjects [9], suggesting that the basement membranes were not fully formed at the time (1 month after grafting) that the grafts were excised. Therefore, further investigation will be required to determine if basement membranes truly develop in vivo. Furthermore, although we have described some of the physical properties of ICX-SKN and its intermediates, ICX-SK N is not fully characterized as to its physiological, biochemical and physical features. The degree of characterization presented here is, however, exactly consistent with the requirements expected of a product at this stage of its clinical development.

The uses of such a product include skin applications such as skin graft following excision of skin cancers (mostly basal and squamous cell carcinomas), following loss of skin volume due to trauma or disease, burns and scar revision. Applying epidermal keratinocytes to the collagen construct and allowing cornification to occur in stu could provide further strength, rigidity, and function, particularly in large-area defects.

\section{Conclusion}

The current ICX-SKN application has been designed as a platform product suitable for use in human clinical trials as a dermal replacement. A four-stage discontinuous manufacturing process provides convenience to both the manufacturer and the end-user and results in a product that is intended to be available off-the-shelf and 


\section{Figure 6. Preclinical study of ICX-SKN skin graft replacement.}

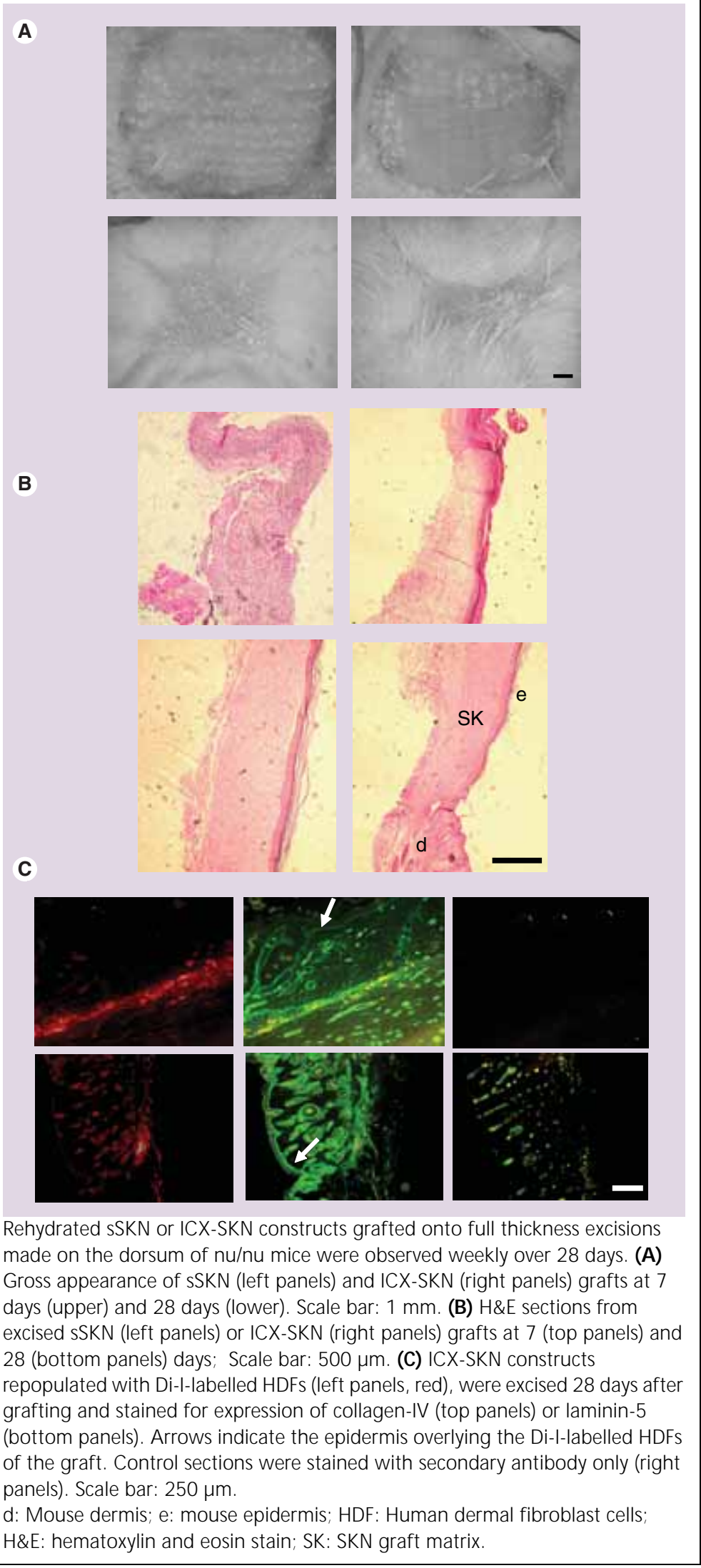

to provide ease-of-use to the clinician. At each stage of its development and manufacture, ICXSKN and its intermediates have been assessed for characteristics that include functional assays indicating its suitability as a clinical trial material. This first iteration of the product lacks barrier function and is intended to be used as a dermal template under conditions where the patient's own epidermis would grow over the dermal graft, such as small excision wounds [9], and to provide contour where tissue has been lost due to trauma or disease. $\mathrm{H}$ owever, we have shown in the current study through the application of keratinocytes and using preclinical models, that this product may eventually be applied as a fully bilayered skin substitute. In addition, the current study suggests the potential for this platform to be used in further indications by the application of different functional cell types.

\section{Future perspective}

ICX-SKN skin graft replacement has been designed with the end-user in mind: the end-user expects an efficacious product but also desires a product that will be easy to use, without complicated preparative steps prior to its application, and off-the-shelf availability. Even in its current iteration, ICX-SKN is a complex product with a long, multistep manufacturing cycle. Marrying the expectations of the customer with the practicalities of manufacturing a living product is one of the continuing challenges for regenerative medicine.

Further development of ICX-SKN and products like it will require input of expertise from many disciplines, particularly engineering, to sustain the scaleup and scale-out that will be necessary to commercialize cell-based therapeutic products. H owever, engineering must understand and accommodate the particular demands of making, storing and shipping a living product such that the essential characteristics that provide the therapeutic benefit are maintained. In addition, the manufacturer of these living products will need to negotiate, and perhaps drive, the complex regulations that govern the use in humans of these therapies and applications.

We have described a manufacturing cycle that is very much based in developments made in the $R \& D$ environment. O ne of the hallmarks of regenerative medicine is that throughout the investigational use of this platform, and its derivatives, the product and the process will be subject to iterative cycles of development. With each iteration, manufacture must be made more costeffective and streamlined without compromising 


\section{Figure 7. Process flow of ICX-SKN production.}

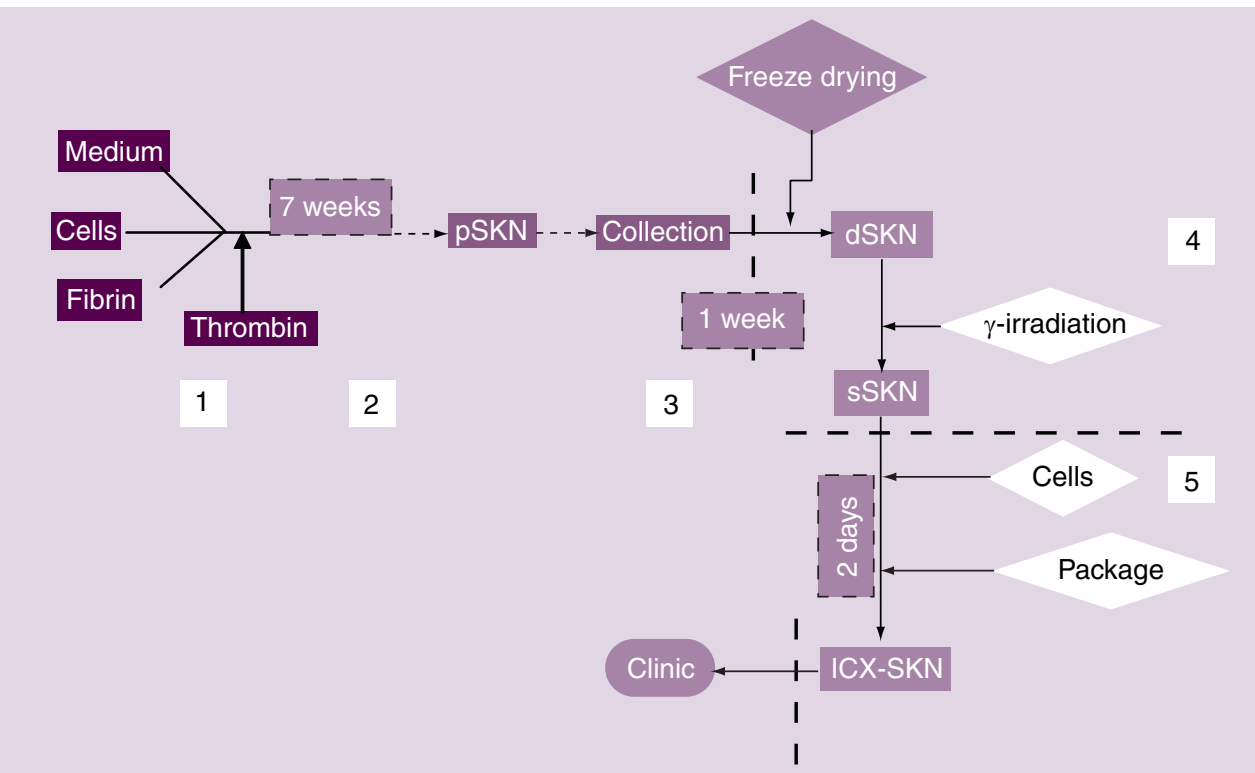

Stages of production are marked by numbers and points of exit/entry to GMP are indicated by dashed lines. Casting of the constructs within GM P facility occurs at stage 1: Reagents are chilled, mixed sequentially and cast immediately upon the addition of thrombin as described ( $M$ aterials and $M$ ethods). M aturation occurs at stage 2, where constructs are fed three times per week with TM for 7 weeks. Matured constructs (pSKN) are freeze dried to preserve the matrix (stage 3 - dSKN), sterilized (stage 4 - sSKN) within 1 week postcollection of pSKN, and finally reintroduced to GMP to be repopulated with HDF (stage 5 - ICX-SKN) over 2 days and packaged, producing the final product for use in the clinic.

GMP: Good manufacturing practice; HDF: Human dermal fibroblast cells; TM: Collagen-boosting medium.

on safety or value to the customer. Effective research translation depends on the realization that a research concept has a long journey before it is suitable for clinical application.

Finally, for a product to be successful will require solution of the three-way equation what science can achieve, what the regulations allow and what the market will bear.

\section{In memoriam}

D avid Shering passed away on 0 ctober 27th 2007 from a sudden illness. He was 29. He made a major contribution to the development of ICX-SKN and the rest of the team would like to dedicate this paper to his memory. $\mathrm{H}$ e will be sorely missed.

\section{Acknowledgements}

We would like to acknowledge our colleagues at Intercytex, past and present, for their contributions to the development and manufacture of ICX-SKN .

Financial \& competing interests disclosure All authors apart from D M arshall are current employees of Intercytex and assuch hold either stock or stock optionsin the Company. The authors have no other relevant affiliations or financial involvement with any organization or entity with a financial interest in or financial conflict with the subject matter or materials discussed in the manuscript apart from those discl osed.

$\mathrm{N}$ o writing assistance was utlilized in the production of this manuscript.

\section{Executive summary}

ICX-SKN is a platform human dermal matrix autosynthesized by human dermal fibroblasts initially embedded in a provisional fibrin matrix.

Discontinuous manufacturing process was designed to provide ready inventory for off-the-shelf availability and ease-of-use for the end user.

Dermal matrix platform remained cell friendly through several intermediate processes in the manufacturing process and was well integrated in host models in vivo.

Potential for this application to be further developed with the addition of keratinocytes to form a fully bilayered product skin equivalent. 


\section{Ethical conduct of research}

The author states that they have obtained appropriate institutional review board approval or have followed the principles outlined in the Declaration of $\mathrm{Helsinki}$ for all human or animal experimental investigations. In addition, for investigations involving human subjects, informed consent has been obtained from the participants involved.

\section{Bibliography}

1. M araguchi $T, M$ araguchi $Y$, Suzuki $S$, M atsuda K, Toda K, Isshiki N : A new skin equivalent: keratinocytes proliferated and differentiated on collagen sponge containing fibroblast. Plast. Reconstr. Surg. 93, 537-44 (1994).

2. Boyce $\mathrm{S}, \mathrm{H}$ ansbrough J: Biologic attachment, grow and differentiation of cultured human epidermal keratinocytes on a graftable collagen and chondroitin6-sulfate substrate. Surgery 103, 421-431 (1988).

3. Parenteau N, Sobolincki M, Prosky S et al.: Biological and physical factors influencing the successful engraftment of a cultured human skin equivalent. Biotechnol. Bioeng. 52, 3-14 (1996).

4. $\mathrm{M}$ acN eil S: Progress and opportunities for tissue-engineered skin. $\mathrm{N}$ ature $445,874-80$ (2007).

5. Ehrenreich M, Ruszczak Z: U pdate on tissue-engineered biological dressings. Tissue Eng. 12, 2407-2424 (2006).

6. Ehrenreich M, Ruszczak Z: U pdate on dermal substitutes. Acta $D$ ermatovenerol. Croat. 14, 172-187 (2006).

7. Tuan $T L$, Song A, Chang S, Younai $S$, $\mathrm{N}$ imni M E: In vitro fibroplasia: matrix contraction, cell growth, and collagen production of fibroblasts cultured in fibrin gels. Exp. Cell Res. 223, 127-134 (1996).

8. N eidert M R, LeeES, 0 egema TR, Tranquillo RT: Enhanced fibrin remodeling in vitro with TGF- $\beta 1$, insulin and plasmin for improved tissueequivalents. Biomaterials 23, 3717-3731 (2002).

9. Boyd M, Flasza M , Johnson PA, St Clair Roberts J, Kemp P: Integration and persistence of an investigational human living skin equivalent (ICX-SKN) in human surgical wounds. Regen. M ed. 2, 363-370 (2007).

10. Rules and guidance for pharmaceutical manufacturers and distributors ( 0 range guide): Pharmaceutical Press, London, UK (2007).

11. Takashima A: Establishment of Fibroblast Cultures. In: Current Protocols in Cell Biology, John Wiley \& Sons, Inc., NY, USA (1998).

12. Reinwaldt JG, Green $\mathrm{H}$ : Serial cultivation of strains of human epidermal keratinocytes: the formation of keratinizing colonies from single cells. Cell 6, 331-344 (1975).

13. M ethods of preparation of sterile products. European Pharmacopoeia, 5th Edition (2007).

14. O Ide D amink LH , Dijkstra PJ, Van Luyn M J, Van Wachem PB, N ieuwenhuis P, Feijen J: Influence of ethylene oxide gas treatment on the in vitro degradation behavior of dermal sheep collagen. J. Biomed. M ater. Res. 29, 149-155 (1995).

15. Cheung DT, Perelman N, Tong D, $\mathrm{N}$ imni M E: The effect of gamma-irradiation on collagen molecules, isolated $\alpha$-chains and crosslinked native fibers. J. Biomed. M ater. Res. 24, 581-589 (1990).

16. Gohari $\mathrm{S}, \mathrm{G}$ ambla $\mathrm{C}, \mathrm{H}$ ealey $\mathrm{M}$ et al.: Evaluation of Tissue-engineered skin (H uman Skin Substitute) and Secondary intention healing in the treatment of full thickness wounds after mohs mictrographic or excisional surgery. D ermatol. Surg. 28, 1107-1113 (2002).

17. Griffiths $M, O$ jeh $N$, Livingstone $R$ et al.: Survival of apligraf in acute human wounds. Tissue Eng. 10, 1180-1196 (2004).

Website

101. Smith \& N ephew: www. wound.smithnephew.com/dermagraft

Patent

201. M urphy M P, Ronfard V: Bioengineered tissue constructs and methods for producing and using thereof. US Patent application, US20020172705 (2002). 